\title{
Levantamento dos hábitos de vida e fatores associados à ocorrência de câncer de tabagistas do município de Sidrolândia (MS, Brasil)
}

\author{
Survey of the life-styles and factors associated with the occurrence \\ of cancer in smokers from the city of Sidrolândia (MS, Brazil)
}

Renata Cristina Losano Feitosa ${ }^{1}$

Elenir Rose Jardim Cury Pontes ${ }^{1}$

${ }^{1}$ Universidade Federal de Mato Grosso do Sul. Cidade Universitária $s / n^{\circ}$. 79070900 Campo Grande MS. renatafeitosa@brturbo.com.br

\begin{abstract}
The study refers to a survey of the lifestyles and factors associated with the occurrence of cancer in smokers of Sidrolândia (MS, Brazil). Study participants were 501 smokers registered in the Family Health Program, which were identified by community health agents. They answered a questionnaire based on the surveys used by the National Institute of Cancer and Prevention and Surveillance Program. The results showed that: the subjects are mostly women from 26 to 45 years of age $(p=0,035)$, living with a partner, do not work out, have little schooling and per capita family income of up to one minimum wage. Also they began to smoke in adolescence and smoke industrialized cigarette. They smoke an average of ten cigarettes a day, even indoors, even knowing that this is harmful to health, exposing the family to passive smoking. As for health problems, 30.6\% have respiratory problems/allergy, $17.4 \%$ high-pressure and $10.8 \%$ depression. The sedentary was reported by $77.6 \%$ of the interviewees, the frequent habit of drinking alcohol by $48.7 \%$ and over-weight by $37.1 \%$. Only $5.8 \%$ of respondents reported only smoking, $94.2 \%$ presented one or more of these factors associated with the occurrence of cancer.
\end{abstract}

Key words Smoking, Life-style, Cancer
Resumo O estudo refere-se a um levantamento dos hábitos de vida e fatores associados à ocorrência de câncer de tabagistas de Sidrolândia (MS). Participaram 501 tabagistas cadastrados no Programa de Saúde da Família, que foram identificados pelos agentes comunitários de saúde. Responderam a um formulário baseado nos inquéritos utilizados pelo Instituto Nacional de Câncer e Programa de Prevenção e Vigilância. Os resultados coletados demonstraram que os sujeitos deste estudo são em sua maioria mulheres de 26 a 45 anos de idade ( $p=0,035)$, residem com companheiro, não trabalham fora, têm pouca escolaridade e renda familiar per capita de até um salário mínimo. Começaram a fumar na adolescência e fazem de uso de cigarro industrializado. Fumam uma média de dez cigarros por dia, inclusive dentro de casa, mesmo sabendo que isso faz mal à saúde, expondo a família ao tabagismo passivo. Quanto aos problemas de saúde, 30,6\% têm problemas respiratórios/ alérgicos; $17,4 \%$, pressão alta; e 10,8\%, depressão. O sedentarismo foi relatado por $77,6 \%$ dos entrevistados; o hábito frequente de ingerir bebidas alcoólicas, por 48,7\%; e o excesso de peso, por $37,1 \%$. Apenas 5,8\% dos entrevistados relataram somente tabagismo; 94,2\% apresentaram, além do tabagismo, um ou mais desses fatores associados à ocorrência de câncer.

Palavras-chave Tabagismo, Hábitos de vida, Câncer 


\section{Introdução}

O câncer é uma doença que afeta não apenas o indivíduo, mas toda a sociedade, por meio da redução do potencial de trabalho humano (per$\mathrm{da}$ de produtividade devido aos adoecimentos e mortes, aposentadorias precoces e pensões) e do impacto econômico resultante dos elevados custos envolvidos com assistência à saúde, poluição, degradação ambiental, pesquisa e educação. Porém, existem gastos imensuráveis como a dor e o sofrimento do doente e da família ${ }^{1}$.

De acordo com o Instituto Nacional de Câncer (Inca), o câncer do pulmão é a maior causa de mortalidade entre os tipos de câncer no Brasil. Também é o tumor maligno mais comum, e sua incidência mundial está a aumentar a uma taxa de $2 \%$ ao ano. Em 90\% dos casos diagnosticados, é associado ao consumo de tabaco ${ }^{2}$.

O hábito de fumar pode aumentar o risco de desenvolver câncer de pulmão em 20 a 30 vezes em tabagistas de longa data, e em 30 a $50 \%$ em fumantes passivos. Não existe, portanto, nenhuma dose ou quantidade segura para o consumo. O estado de Mato Grosso do Sul tem uma taxa estimada de 19 casos de câncer de traqueia, brônquios e pulmão para cada 100 mil homens e dez casos para cada 100 mil mulheres. Para os homens, o câncer de pulmão é o segundo mais frequente; para as mulheres, o câncer de pulmão é o quarto tipo mais frequente ${ }^{3}$.

De acordo com o estudo de Oliveira et al. ${ }^{4}$, cerca de $10 \%$ dos tumores malignos que ocorrem no corpo humano localizam-se na boca, sendo o câncer bucal o sexto tipo de câncer mais incidente no mundo, podendo ser considerado o mais comum (com exceção do câncer de pele) na região da cabeça e pescoço. Destaca-se que o tabaco e o álcool são os dois fatores de risco mais importantes, não só para o desenvolvimento da neoplasia como também para seu prognóstico. No estudo desses autores, $68,5 \%$ dos pacientes $(n=340)$ eram tabagistas e etilistas.

O consumo de bebidas alcoólicas aumenta cerca de nove vezes o risco de câncer de boca, e quando associado ao tabagismo esse risco torna-se 35 vezes maior. Também está associado a um aumento de $50 \%$ no risco de câncer de fígado. Entre mulheres, o risco de câncer de mama pode ser $60 \%$ maior entre as que consomem 15 gramas ou mais de etanol por dia do que entre as que não consomem bebidas alcoólicas. Ao consumo excessivo de álcool são atribuídas de $2 \%$ a $4 \%$ das mortes por câncer e, mais especificamente, de $50 \%$ a $70 \%$ de todas as mortes por câncer de língua, cavidade oral, faringe e esôfago ${ }^{5}$.
Uma alimentação pobre em fibras, com altos teores de gordura e calóricas (hambúrguer, batata frita, bacon etc.), está relacionada a um maior risco para o desenvolvimento de câncer de cólon, de reto, de mama e de próstata. Esses tipos de câncer são mais comuns em regiões mais desenvolvidas, principalmente no Ocidente, onde há alto consumo de alimentos ricos em gorduras (fast food). Já os cânceres de estômago e de esôfago ocorrem mais frequentemente em alguns países do Oriente e em regiões pobres, onde não há meios adequados de conservação dos alimentos (geladeira), o que torna comum o uso de picles e alimentos preservados em sal, como peixes e carnes salgadas 5 .

A prática regular de atividade física reduz o risco de mortes prematuras, doenças cardíacas, cânceres e diabetes tipo II, além de prevenir ou reduzir a hipertensão arterial, a obesidade e a osteoporose. O condicionamento físico também reduz a mortalidade e a morbidade, mesmo em pessoas que se mantêm obesas ${ }^{6}$.

O câncer de pele é causado principalmente pela exposição excessiva ao sol. Os raios ultravioletas (UV) podem ser classificados em UVA, UVB e UVC. Os raios UVB são responsáveis por queimaduras solares, câncer de pele e lesões destrutivas das camadas mais profundas da pele, que levam ao envelhecimento cutâneo. Os raios UVA penetram profundamente na pele e causam alterações que contribuem para o envelhecimento precoce e potencializam o efeito carcinogênico dos raios UVB. Os raios UVC não alcançam a superfície da terra, não tendo, portanto, efeitos sobre a pele. O filtro natural que absorve na atmosfera os raios UVB e UVC é a camada de ozônio. Os raios UVA não são absorvidos por essa camada, atingindo plenamente a superfície do planeta. Devido à destruição da camada de ozônio, maior frequência de casos de câncer de pele vêm ocorrendo, principalmente nos países próximos ao Equador, como é o caso do Brasil ${ }^{5}$.

Muitas substâncias químicas usadas na indústria podem constituir um fator de risco de câncer em trabalhadores de diversas ocupações. Quando, além desse fator, o trabalhador também é fumante, o risco torna-se ainda maior, pois o fumo pode interagir com a capacidade cancerígena de muitas substâncias ${ }^{7}$.

A precocidade do início da vida sexual (antes dos 18 anos), a promiscuidade sexual e a falta de higiene estão relacionadas a um maior risco de câncer do colo uterino. Assim, os hábitos sexuais contribuem para a propagação de agentes sexualmente transmissíveis que podem induzir ao câncer, como o herpes vírus II e o papiloma vírus 
humano (HPV), que estão relacionados com o câncer do colo uterino, o vírus HTLV-I, associado às leucemias, e o vírus da hepatite $B$, que se relaciona com o câncer de fígado ${ }^{5}$.

De acordo com o Inca, $80 \%$ dos casos de câncer estão relacionados ao meio ambiente em geral: o ambiente ocupacional (indústrias químicas e afins), o ambiente de consumo (alimentos, medicamentos) e o ambiente social e cultural (estilos e hábitos de vida). Desta forma, as mudanças no meio ambiente (provocadas pelo próprio homem), os hábitos e o estilo de vida atual das pessoas em geral podem determinar diferentes tipos de câncer8.

Este trabalho teve por objetivo realizar um levantamento sobre os hábitos de vida e os principais fatores associados à ocorrência de câncer de tabagistas do município de Sidrolândia (MS), cadastrados no Programa Saúde da Família (PSF) - atual Estratégia Saúde da Família.

\section{Método}

Este estudo é transversal, descritivo e de abordagem quantitativa. Foi desenvolvido no município de Sidrolândia (MS), que possui uma área de $5.300,9$ quilômetros quadrados, localizado a 64 quilômetros de Campo Grande, capital de Mato Grosso do Sul. A população do município, segundo estimativa do Instituto Brasileiro de Geografia e Estatística (IBGE) de julho de 2005, era de 28.412 habitantes, sendo que um terço residia em zona rural; porém este número é crescente, devido ao grande número de acampamentos e assentamentos rurais que se formam no município constantemente. A economia do município baseia-se principalmente na agropecuária, porém tem-se destacado nos últimos anos pelo grande crescimento da atividade industrial e comercial, o que também atrai novos moradores.

Em 2006, o município contava na área urbana com três equipes do Programa Saúde da Família, com uma cobertura de atendimento de $68 \%$ (12.812 pessoas de uma população urbana de aproximadamente 18.941 habitantes), correspondendo à proporção de uma equipe para cada 1.177 famílias. Foram sujeitos deste estudo todos os tabagistas cadastrados nessas equipes.

Os tabagistas foram identificados durante a visita domiciliar mensal dos agentes comunitários de saúde do PSF no mês de maio de 2006, que registraram na Ficha A, no campo "doença ou condição referida”, as siglas: TAB - F (tabagista fumante), $\mathrm{TAB}-\mathrm{N}$ (tabagista que naqueia) ou
TAB - FN (tabagista fumante e que naqueia), totalizando 597 tabagistas.

Como instrumento de coleta de dados, foi utilizado um formulário envolvendo questões que permitiram levantar dados sobre os hábitos de vida dos tabagistas do município. As questões do formulário foram baseadas nos questionários utilizados pelo Instituto Nacional de Câncer, no Programa Nacional de Controle do Tabagismo e Outros Fatores de Risco de Câncer (PNCT) e Coordenação de Prevenção e Vigilância (Conprev).

Os formulários foram aplicados pelos agentes comunitários de saúde do município após treinamento por uma das pesquisadoras deste estudo, que os acompanhou nas visitas até que estivessem aptos para a correta abordagem dos entrevistados e o registro fidedigno das informações obtidas.

Os resultados do trabalho foram submetidos à análise estatística descritiva e analítica. Para verificar possíveis associações entre as variáveis de estudo, foi utilizado o teste Qui-quadrado ao nível de significância de 5\%. Foi utilizado o programa Epi-Info versão 3.2.2. A pesquisa foi submetida ao Comitê de Ética da Universidade Federal de Mato Grosso do Sul, tendo sido seguidos todos os procedimentos padrão.

\section{Resultados}

Do total de 597 tabagistas, 501 participaram da pesquisa. A não participação dos outros 96 tabagistas foi devido aos seguintes motivos: oito se recusaram a participar do estudo; 31 não foram encontrados na residência após três visitas do agente comunitário de saúde, sendo na maioria homens trabalhadores da zona rural; 36 pessoas mudaram da área de cobertura do Programa de Saúde da Família e 21 pessoas haviam deixado de fumar. Quanto às características sociodemográficas dos sujeitos deste estudo $(n=501)$, pode-se observar que $42,9 \%$ são adultos compreendendo a faixa etária de 26 a 45 anos, 55,7\% são mulheres, 66,6\% não possuem o ensino fundamental completo, $12,4 \%$ são analfabetos e 95,8\% não estudam atualmente. Sobre a situação conjugal, 67,3\% dos participantes têm companheiro(a) e 93,2\% residem com a família. A maioria dos entrevistados $(92,2 \%)$ possui uma renda familiar per capita de um salário mínimo, 30,9\% não possuem uma ocupação profissional e 13,4\% possuem ocupações profissionais consideradas de risco, pois há exposição a materiais de pintura, solvente, agrotóxicos, inseticidas, fuligem de madeira, de grãos ou de tecido. 
Dentre os 501 tabagistas que participaram da pesquisa, $23(4,6 \%)$ apenas naqueiam e $17(3,4 \%)$ fumam e naqueiam, totalizando quarenta tabagistas que naqueiam (8\%). Desses que naqueiam, $20 \%$ adquiriram este hábito na faixa etária até 10 anos e 50\% entre 11 e 20 anos. Quanto à frequência, $45 \%$ naqueiam quatro vezes ou mais ao dia.

Do total de tabagistas fumantes $(n=478)$, apenas $227(47,5 \%)$ trabalham fora. Destes, 19 $(8,4 \%)$ fumam no local de trabalho (ambiente fechado). Na Tabela 1 pode-se observar a distribuição desses fumantes em relação à idade em que começaram a fumar, tipo e quantidade de cigarros que fumam diariamente e o hábito de fumar dentro de casa.

No grupo estudado $(n=501), 50,5 \%$ dos indivíduos disseram que possuem algum problema de saúde e estes estão diretamente associados ao tabagismo. Os principais problemas citados foram: os respiratórios $(20 \%)$, a hipertensão $(17,4 \%)$, a depressão $(10,8 \%)$ e os alérgicos $(10,6 \%)$. A grande maioria dos entrevistados $(92,8 \%)$ acredita que o tabagismo faz mal à saúde, e $86,2 \%$ têm o desejo de deixar de ser tabagis- ta. Na Tabela 2 pode-se visualizar o grau de índice de massa corpórea (IMC), hábito de fazer dieta, consumo de carne gorda, salada e hábito de praticar atividade física, e na Tabela 3, o etilismo.

Em relação à exposição solar, de 222 homens, $36 \%$ relataram que sempre se expõem ao sol no período das $10 \mathrm{~h} 30 \mathrm{~min}$ às $16 \mathrm{~h} 30 \mathrm{~min}$, por mais de trinta minutos, sendo que de 279 mulheres, $16,8 \%$ fazem isso $(p<0,001)$. No sexo masculino, $82 \%$ nunca usam protetor solar, e no sexo feminino, a porcentagem é de $56 \%(p<0,001)$. Do total de 501 entrevistados, $67 \%$ disseram que a exposição ao sol ocorre nos locais de trabalho ou na locomoção.

Quanto ao contato com produtos químicos no trabalho, de 501 tabagistas, $12,8 \%(n=64)$ estão expostos; destes, $40,6 \%$ não usam equipamentos de proteção individual (EPI).

Sobre as doenças sexualmente transmissíveis (DST), 90\% das mulheres $(\mathrm{n}=279)$ responderam que nunca tiveram. Das 28 mulheres que tiveram DST, a de maior ocorrência foi a candidíase. Apenas três mulheres tiveram HPV (papiloma vírus humano).

Tabela 1. Distribuição dos tabagistas usuários do SUS (exceto quem somente naqueia), segundo sexo e variáveis de estudo - Sidrolândia (MS), 2006.

\begin{tabular}{|c|c|c|c|c|c|c|c|}
\hline \multirow[t]{2}{*}{ Variáveis } & \multicolumn{2}{|c|}{$\begin{array}{c}\text { Masculino } \\
(\mathrm{n}=203)\end{array}$} & \multicolumn{2}{|c|}{$\begin{array}{c}\text { Feminino } \\
(\mathrm{n}=275)\end{array}$} & \multicolumn{2}{|c|}{$\begin{array}{c}\text { Total } \\
(n=478)\end{array}$} & \multirow{2}{*}{$p$} \\
\hline & $\mathbf{N}^{\circ}$ & $\%$ & $\mathbf{N}^{\circ}$ & $\%$ & $\mathbf{N}^{\circ}$ & $\%$ & \\
\hline \multicolumn{8}{|l|}{ Idade } \\
\hline Até 5 anos & - & - & 3 & 1,1 & 3 & 0,6 & 0,060 \\
\hline De 6 a 10 anos & 38 & 18,7 & 30 & 10,9 & 68 & 14,2 & \\
\hline De 11 a 15 anos & 73 & 36,0 & 123 & 44,7 & 196 & 41,0 & \\
\hline De 16 a 20 anos & 70 & 34,5 & 94 & 34,2 & 164 & 34,3 & \\
\hline De 21 a 30 anos & 19 & 9,3 & 19 & 6,9 & 38 & 8,0 & \\
\hline Mais de 31 anos & 3 & 1,5 & 6 & 2,2 & 9 & 1,9 & \\
\hline \multicolumn{8}{|l|}{ Tipo de cigarro } \\
\hline Cigarro industrializado & 97 & 47,8 & 182 & 66,2 & 279 & 58,4 & ${ }^{*}<0,001$ \\
\hline Cigarro de fumo (palha/papel) & 36 & 17,7 & 44 & 16,0 & 80 & 16,7 & \\
\hline Cigarro industrializado e fumo & 67 & 33,0 & 48 & 17,4 & 115 & 24,1 & \\
\hline Charuto ou cachimbo & 3 & 1,5 & 1 & 0,4 & 4 & 0,8 & \\
\hline \multicolumn{8}{|l|}{ Quantidade de cigarros por dia (UN) } \\
\hline Até 10 cigarros & 79 & 38,9 & 158 & 57,4 & 237 & 49,6 & $<0,001$ \\
\hline De 11 a 20 cigarros & 90 & 44,3 & 100 & 36,4 & 190 & 39,7 & \\
\hline De 21 a 30 cigarros & 14 & 6,9 & 6 & 2,2 & 20 & 4,2 & \\
\hline Mais de 31 cigarros & 20 & 9,9 & 11 & 4,0 & 31 & 6,5 & \\
\hline \multicolumn{8}{|l|}{ Hábito de fumar dentro de casa } \\
\hline Sim & 127 & 62,6 & 186 & 67,6 & 313 & 65,5 & ${ }^{* *} 0,291$ \\
\hline Não & 76 & 37,4 & 89 & 32,4 & 165 & 34,5 & \\
\hline
\end{tabular}

Nota: Se $p \leq 0,05$ - diferença estatisticamente significativa. Teste Qui-quadrado.

"Foi suprimida a categoria "charuto ou cachimbo" para cálculo do Qui-quadrado; ${ }^{* *}$ Teste Qui-quadrado corrigido por Yates. 
Tabela 2. Distribuição dos tabagistas usuários do SUS, segundo sexo e variáveis referentes à nutrição e prática de atividade física - Sidrolândia (MS), 2006.

\begin{tabular}{|c|c|c|c|c|c|c|c|}
\hline \multirow[t]{2}{*}{ Variáveis } & \multicolumn{2}{|c|}{$\begin{array}{c}\text { Masculino } \\
(\mathrm{n}=22)\end{array}$} & \multicolumn{2}{|c|}{$\begin{array}{r}\text { Feminino } \\
(\mathrm{n}=279)\end{array}$} & \multicolumn{2}{|c|}{$\begin{array}{c}\text { Total } \\
(n=501)\end{array}$} & \multirow{2}{*}{$p$} \\
\hline & $\mathbf{N}^{\circ}$ & $\%$ & $\mathbf{N}^{\circ}$ & $\%$ & $\mathbf{N}^{\circ}$ & $\%$ & \\
\hline \multicolumn{8}{|l|}{ Grau de IMC } \\
\hline Baixo peso (menos de 18,5 ) & 8 & 3,6 & 16 & 5,7 & 24 & 4,8 & 0,267 \\
\hline Eutrófico (de 18,5 a 24,999) & 135 & 60,8 & 156 & 55,9 & 291 & 58,1 & \\
\hline Sobrepeso (de 25 a 29,999) & 60 & 27,0 & 71 & 25,5 & 131 & 26,1 & \\
\hline Obesidade (acima de 29,999) & 19 & 8,6 & 36 & 12,9 & 55 & 11,0 & \\
\hline \multicolumn{8}{|l|}{ Costuma fazer dieta } \\
\hline Sim & 22 & 9,9 & 60 & 21,5 & 82 & 16,4 & ${ }^{*}<0,001$ \\
\hline Não & 200 & 90,1 & 219 & 78,5 & 419 & 83,6 & \\
\hline \multicolumn{8}{|l|}{ Consumo de carne gorda } \\
\hline Sempre & 89 & 40,1 & 88 & 31,5 & 177 & 35,3 & 0,013 \\
\hline Às vezes & 68 & 30,6 & 73 & 26,2 & 141 & 28,1 & \\
\hline $\begin{array}{l}\text { Raramente } \\
\text { Nunca }\end{array}$ & 29 & 13,1 & 41 & 14,7 & 70 & 14,0 & \\
\hline Consumo de salada & 36 & 16,2 & 77 & 27,6 & 113 & 22,6 & \\
\hline \multicolumn{8}{|l|}{ Sempre } \\
\hline Às vezes & 117 & 52,7 & 194 & 69,5 & 311 & 62,1 & 0,001 \\
\hline Raramente & 66 & 29,7 & 57 & 20,4 & 123 & 24,5 & \\
\hline Nunca & 30 & 13,5 & 20 & 7,2 & 50 & 10,0 & \\
\hline $\begin{array}{l}\text { Costuma praticar atividade física } \\
\text { como lazer ou exercício físico }\end{array}$ & 9 & 4,1 & 8 & 2,9 & 17 & 3,4 & \\
\hline Sempre & 41 & 18,4 & 18 & 6,5 & 59 & 11,8 & $<0,001$ \\
\hline Às vezes & 25 & 11,3 & 28 & 10,0 & 53 & 10,6 & \\
\hline Raramente & 25 & 11,3 & 28 & 10,0 & 53 & 10,6 & \\
\hline Nunca & 131 & 59,0 & 205 & 73,5 & 336 & 67,0 & \\
\hline
\end{tabular}

Nota: Se $p \leq 0,05$ - diferença estatisticamente significativa. Teste Qui-quadrado.

*Teste Qui-quadrado corrigido por Yates.

Tabela 3. Distribuição dos tabagistas usuários do SUS segundo sexo e etilismo - Sidrolândia (MS), 2006.

\begin{tabular}{|c|c|c|c|c|c|c|c|}
\hline \multirow[t]{2}{*}{ Variáveis } & \multicolumn{2}{|c|}{$\begin{array}{c}\text { Masculino } \\
(\mathrm{n}=222)\end{array}$} & \multicolumn{2}{|c|}{$\begin{array}{r}\text { Feminino } \\
(\mathrm{n}=279)\end{array}$} & \multicolumn{2}{|c|}{$\begin{array}{c}\text { Total } \\
(n=501)\end{array}$} & \multirow{2}{*}{$p$} \\
\hline & $\mathbf{N}^{\circ}$ & $\%$ & $\mathrm{~N}^{\circ}$ & $\%$ & $\mathbf{N}^{\circ}$ & $\%$ & \\
\hline \multicolumn{8}{|l|}{ Hábito de tomar bebida alcoólica } \\
\hline Sempre & 46 & 20,7 & 29 & 10,4 & 75 & 15,0 & 0,005 \\
\hline Às vezes & 73 & 32,9 & 96 & 34,4 & 169 & 33,7 & \\
\hline Raramente & 39 & 17,6 & 45 & 16,1 & 84 & 16,8 & \\
\hline Nunca & 64 & 28,8 & 109 & 39,1 & 173 & 34,5 & \\
\hline \multicolumn{8}{|c|}{ Necessidade de tomar bebida alcoólica } \\
\hline Não bebem & 64 & 28,8 & 109 & 39,1 & 173 & 34,5 & 0,001 \\
\hline Sim, todos os dias & 21 & 9,5 & 7 & 2,5 & 28 & 5,6 & \\
\hline Sim, só nos fins de semana & 57 & 25,7 & 56 & 20,1 & 113 & 22,6 & \\
\hline Não & 80 & 36,0 & 107 & 38,3 & 187 & 37,3 & \\
\hline
\end{tabular}

Nota: Se $p \leq 0,05$ - diferença estatisticamente significativa. Teste Qui-quadrado. 
Quanto à realização de exame preventivo ginecológico, $87,8 \%$ das mulheres $(n=279)$ referiram que já fizeram e 11,1\% nunca fizeram. Considerando o ano em que foi realizado o exame, percebe-se que são recentes, visto que $64,5 \%$ das mulheres fizeram em 2005 ou 2006.

Do total de 279 mulheres, $73,5 \%$ fazem uso de métodos contraceptivos, sendo que 39,4\% realizaram laqueadura (ligadura de trompas), 19\% usam pílula e $14 \%$ usam preservativo masculino.

Dos homens participantes deste estudo $(\mathrm{n}=222), 89,2 \%$ referiram que nunca tiveram DST. Dos que tiveram alguma DST $(n=24)$, a candidíase foi a mais frequente, sendo que apenas dois homens não fizeram tratamento algum. Apenas 29,3\% dos homens têm o hábito de sempre usar preservativo nas relações sexuais e 48,6\% nunca usam. Quanto aos homens com mais de 50 anos de idade ( $n=75), 53,3 \%$ não fizeram o exame da próstata (PSA).

Considerando os principais fatores associados à ocorrência de câncer - sedentarismo, consumo frequente de álcool e índice de massa corporal acima do normal, buscou-se na Tabela 4 verificar na população de estudo a presença desses fatores além da condição de tabagista.

\section{Discussão}

Segundo estudos da Organização Mundial da Saúde, tabagismo, pobreza e baixa escolaridade estão relacionados. Na China, a pessoa de baixa escolaridade tem cerca de sete vezes mais probabilidade de ser fumante do que pessoas que têm o terceiro grau. No Brasil, esta probabilidade é de cinco vezes ${ }^{9}$.

Tabela 4. Distribuição dos tabagistas usuários do SUS segundo a presença de fatores associados à ocorrência de câncer - Sidrolândia (MS), 2006.

\begin{tabular}{lrr}
\hline \multicolumn{1}{c}{ Fatores } & \multicolumn{1}{c}{ N $^{\text { }}$} & \multicolumn{1}{c}{$\%$} \\
\hline Apenas tabagista & 29 & 5,8 \\
Tabagista e tem mais um fator associado & 190 & 37,9 \\
Tabagista e tem mais dois fatores associados & 217 & 43,3 \\
Tabagista e tem mais três fatores associados & 65 & 13,0 \\
Total & 501 & 100,0 \\
\hline
\end{tabular}

Nota: para esta análise, foram considerados os fatores associados à ocorrência de câncer - sedentarismo, consumo frequente de álcool e índice de massa corporal acima do normal, além da condição de ser tabagista.
Em relação aos tabagistas fumantes $(\mathrm{n}=478)$ (Tabela 1), 41\% começaram a fumar com idades entre 11 e 15 anos, ou seja, na adolescência, porém chama a atenção que três mulheres $(0,6 \%)$ começaram a fumar com idade até 5 anos e 68 pessoas $(14,2 \%)$ começaram a fumar dos 6 aos 10 anos, ou seja, ainda na infância.

De acordo com o Ministério da Saúde ${ }^{10}$, o consumo de tabaco entre as mulheres vem aumentando, principalmente na faixa etária mais jovem. Sabe-se que um terço da população adulta fuma, sendo 11,2 milhões de mulheres e 16,7 milhões de homens. Destes, $90 \%$ se tornaram dependentes da nicotina entre 5 e 19 anos de idade.

Para Moreira et al. ${ }^{11}$, é fundamental investir em programas de prevenção ao tabagismo para adolescentes, afinal é nesta faixa etária que tem início o hábito de fumar.

Segundo a Tabela 1, o tipo de cigarro mais consumido pelos tabagistas $(\mathrm{n}=478)$ é o industrializado $(58,4 \%)$. Porém, $24,1 \%$ têm o hábito de fumar cigarros industrializados e de fumo (palha), sendo esta combinação mais habitual para os homens $(p=0,0001)-33 \%$ de 203 indivíduos - em relação ao sexo feminino - 17,4\% de 275 mulheres. Quanto à quantidade de cigarros, observa-se que $49,6 \%$ fumam até dez cigarros e $39,7 \%$, de 11 a 20 cigarros por dia.

Mesmo observando que a maioria dos participantes deste estudo consome até dez cigarros por dia, vale ressaltar que não existem níveis seguros de consumo; o fumante de menos de dez cigarros ao dia apresenta seis vezes mais chances de ter câncer de pulmão do que o não fumante. Mesmo os que não tragam têm dez vezes mais chances ${ }^{9}$.

Naquear também oferece prejuízos à saúde. O tabaco mascado deixa resíduos na boca (entre a bochecha e a língua) e favorece, através do contato prolongado, a ação das substâncias cancerígenas do tabaco sobre a mucosa bucal. O tabaco é responsável por cerca de $90 \%$ dos casos de câncer bucal e é prejudicial tanto mascado quanto fumado ou aspirado ${ }^{12}$.

A maioria dos tabagistas fumantes tem o hábito de fumar dentro de casa (Tabela 1). Isto demonstra que o tabagismo passivo é muito comum nessas famílias. Além disso, há os que fumam no local de trabalho, estando assim em desacordo com a Lei $\mathrm{n}^{\circ}$ 9.294/96, que proíbe fumar em ambientes fechados, públicos ou privados.

A fumaça inalada por pessoas que convivem com fumantes em seu local de trabalho durante oito horas diárias provoca malefícios equivalentes ao consumo de seis cigarros por dia. Ficar em 
uma mesma casa, no período de 24 horas, onde se consome cerca de vinte cigarros por dia equivale a ter fumado três cigarros por $\operatorname{dia}^{13}$.

No grupo analisado, a maioria tem problemas de saúde que estão diretamente relacionados ao consumo de tabaco. Chama a atenção o fato de que $10,8 \%$ dos tabagistas relataram que apresentam depressão. Um estudo envolvendo 4 mil homens e 5 mil mulheres (todos gêmeos), realizado na Finlândia através de monitoramento da saúde e do comportamento monitorado por 15 anos, revelou que fumantes de longa data têm mais risco de ficarem depressivos do que aqueles que nunca fumaram. Essa associação não foi verificada em fumantes que deixaram de fumar há muitos anos. Esta pesquisa destacou que quando as pessoas começam a fumar, os efeitos imediatos da nicotina no cérebro são compensadores e agradáveis, o que faz com que muitos fumantes busquem alívio no cigarro, tornandose dependentes da nicotina ${ }^{14}$.

A mais longa pesquisa já realizada pela Universidade de Oxford sobre os efeitos do tabaco, que envolveu 34.439 pessoas, revelou que os fumantes morrem em média dez anos antes dos não fumantes. $\mathrm{O}$ estudo esclarece que os riscos do tabagismo para a saúde são maiores do que se pensava inicialmente, e informa também que deixar de fumar em qualquer idade reduz o risco de morrer por doenças ligadas ao tabagismo ${ }^{15}$.

Os hábitos alimentares podem ser fatores de proteção ou fatores de risco para câncer. Acredita-se que cerca de 35\% dos diversos tipos de câncer ocorrem em razão de dietas inadequadas ${ }^{16}$. Conforme a Tabela 2, a maioria da população estudada tem o hábito de comer sempre ou às vezes carne gorda e churrasco, principalmente os homens. As mulheres, em geral, têm mais costume de comer salada.

Em relação ao índice de massa corpórea (IMC) demonstrado na Tabela 2, a maioria dos participantes está dentro da faixa de peso considerada normal, porém chama a atenção o fato de que $37,1 \%$ estão com sobrepeso ou obesos e a grande maioria não tem o hábito de fazer dieta alimentar, sendo este hábito mais adotado pelas mulheres. A obesidade está relacionada com maior risco de câncer de útero (endométrio), de vesícula biliar, de mama e de cólon ${ }^{5}$.

Quanto à prática de atividade física (Tabela 2), pode-se observar que não é um hábito frequente para os tabagistas, porém os homens praticam mais que as mulheres. A atividade física diminui os riscos de câncer de cólon, mama e pulmão. Além disso, ajuda a manter o equilíbrio entre a ingestão calórica e o gasto energético, evitando o aumento de peso, e desta forma também contribui para a redução dos riscos de doenças cardiovasculares e diabetes ${ }^{17}$.

Pode-se observar que a maioria dos homens costuma sempre se expor ao sol em horário impróprio, no entanto as mulheres ficam menos expostas e apresentam menor frequência das que nunca usam filtro solar. Como a exposição ocorre mais nos locais de trabalho e locomoção, pode ser que o uso do filtro solar esteja mais associado ao lazer, havendo uma negligência no uso diário desses protetores.

Dos tabagistas que se expõem a produtos químicos, muitos não utilizam nenhum tipo de equipamento de proteção individual (EPI), o que pode indicar o contato direto do produto com os lábios, caso fumem durante o período de exposição. O estudo de Ribeiro e Wünsch Filho ${ }^{18}$ alerta que a Organização Mundial da Saúde reconhece 88 agentes ou circunstâncias como cancerígenos para os humanos, dos quais 23 são encontrados principalmente em ambientes ocupacionais e $13 \mathrm{em}$ processos de trabalho.

O câncer provocado pela exposição ocupacional pode atingir as regiões do corpo que estão em contato direto com as substâncias cancerígenas, tanto durante a fase de absorção (pele ou aparelho respiratório) quanto na fase de excreção (aparelho urinário), o que explica a maior frequência de câncer de pulmão, de pele e de bexiga nesse tipo de exposição ${ }^{19}$.

No presente estudo (Tabela 3), os homens ingerem bebida alcoólica com maior frequência e possuem maior necessidade do álcool. As mulheres bebem às vezes com maior necessidade nos fins de semana.

Segundo Smith-Warner et al. ${ }^{20}$, mesmo consumindo a mesma quantidade, existe diferença de absorção do álcool entre homens e mulheres; ele destaca que, além de serem mais sensíveis aos efeitos embriagadores do álcool, as mulheres progridem mais rapidamente para o alcoolismo crônico e suas demais complicações médicas, correndo mais risco de desenvolver doenças hepáticas, cirrose, doenças cardiovasculares, hipertensão arterial, osteoporose e câncer. A metaanálise de seis estudos importantes mostrou que mulheres habituadas a ingerir de 2,5 a cinco drinques por dia apresentaram probabilidade $40 \%$ maior de desenvolver câncer de mama.

O HPV (papiloma vírus humano) está presente em 94\% dos casos de câncer do colo de útero, que é o terceiro câncer que mais mata no Brasil, mesmo sendo uma doença que pode ser 
prevenida através do exame preventivo ginecológico ${ }^{21}$. Apenas três mulheres disseram ter tido HPV, e a realização recente do exame ginecológico foi um relato comum das entrevistadas, o que pode indicar uma preocupação com a prevenção de doenças e autocuidado.

No entanto, observou-se baixa frequência de realização do exame de próstata (PSA) pelos homens com mais de 50 anos. Vale ressaltar que este exame, tão importante no diagnóstico precoce de câncer de próstata, não é disponível na rede pública de saúde.

No estudo de César et al. ${ }^{22}$, o método anticoncepcional mais comum foi o anticoncepcional oral (69\%), seguido por ligadura de trompas (19\%). No presente estudo, pode-se observar uma parcela menor de utilização da pílula e maior frequência de laqueadura, o que de certa forma isenta esta população da exposição aos efeitos colaterais do medicamento; no entanto, isto remete à tomada de decisão consciente em relação à opção por métodos anticoncepcionais definitivos, bem como o uso da camisinha como proteção contra as doenças sexuais transmissíveis.

O hábito de fumar, por si só, já é causa suficiente para a ocorrência de câncer no indivíduo, porém, na presença de outros fatores maléficos à saúde, pode haver a potencialização do risco de desenvolvimento de neoplasias. Segundo a $\mathrm{Ta}$ bela 4 , considerando como fatores associados à ocorrência de câncer o tabagismo, o sedentarismo, o consumo frequente de álcool e o peso corporal acima do normal, apenas $5,8 \%$ dos entrevistados relataram somente tabagismo, $94,2 \%$ apresentaram, além do tabagismo, um ou mais fatores associados.

\section{Colaboradores}

RCL Feitosa realizou a coleta de dados, análise, interpretação e discussão e redação do artigo; ERJC Pontes participou do delineamento metodológico, da discussão dos dados e da redação do artigo.

\section{Conclusão}

Este estudo apresenta dados semelhantes a outros disponíveis na literatura, confirmando que uma parcela expressiva dos tabagistas tem pouca escolaridade, baixa renda e começou a fumar na adolescência. Revela ainda que os tabagistas possuem problemas de saúde relacionados ao uso do tabaco, principalmente os respiratórios e alérgicos, e que apresentam outros fatores associados à ocorrência de câncer, de modo especial o sedentarismo, o uso frequente de bebida alcoólica e o excesso de peso.

Um desdobramento importante desta pesquisa foi a divulgação dos resultados aos agentes comunitários, que passaram a demonstrar maior engajamento na busca ativa de tabagistas, a fim de aumentar a adesão ao Programa Municipal de Controle do Tabagismo e Outros Fatores de Risco de Câncer, desenvolvido pelo município. Este programa oferece acompanhamento psicológico e disponibiliza insumos medicamentosos, que são utilizados como tratamento coadjuvante, para auxiliar os tabagistas a pararem de fumar.

Desta forma, torna-se fundamental que se invista em ações de promoção da saúde e prevenção de doenças, a fim de alertar a comunidade sobre os fatores de risco de câncer. Os que já são tabagistas devem ser orientados a participar do programa de tratamento do fumante, e as crianças e os adolescentes devem ser envolvidos em ações educativas nas escolas e unidades de saúde, a fim de que conheçam os malefícios do tabagismo.

\section{Agradecimentos}

Ao apoio técnico da Coordenação Estadual do Programa Municipal de Controle do Tabagismo e Outros Fatores de Risco de Câncer e da Secretaria Municipal de Saúde de Sidrolândia (MS). 


\section{Referências}

1. Brasil. Ministério da Saúde. Pare de fumar: tabaco e economia. Rio de Janeiro: Inca; 2005.

2. Brasil. Ministério da Saúde. Instituto Nacional de Câncer (Inca). Câncer de pulmão. Rio de Janeiro: Inca; 2006.

3. Brasil. Ministério da Saúde. Secretaria de Atenção à Saúde. Estimativa 2006: incidência de câncer no Brasil. Rio de Janeiro: Inca; 2005.

4. Oliveira LR, Ribeiro-Silva A, Zucoloto S. Perfil da incidência e da sobrevida de pacientes com carcinoma epidermóide oral em uma população brasileira. J Bras Patol Med Lab 2006; 42(5):385-392.

5. Brasil. Ministério da Saúde. Falando sobre câncer e seus fatores de risco. $2^{\text {a }}$ ed. Rio de Janeiro: Ministério da Saúde; 1998.

6. Brasil. Ministério da Saúde. Secretaria de Vigilância em Saúde. Coordenação de Prevenção e Vigilância. Inquérito domiciliar sobre comportamentos de risco e morbidade referida de doenças e agravos não transmissíveis - Fazendo pesquisa: manual do entrevistador. Rio de Janeiro: Inca; 2004.

7. Ribeiro FS, Wünsch Filho V. Avaliação retrospectiva da exposição ocupacional a cancerígenos: abordagem epidemiológica e aplicação em vigilância em saúde. Cad Saude Publica 2004; 20(4):881-890.

8. Brasil. Ministério da Saúde. Fatores de risco. Rio de Janeiro: Inca; 2005.

9. Organização Pan-Americana da Saúde (Opas). Tabaco e pobreza: um círculo vicioso. Brasília: Opas; 2004.

10. Brasil. Ministério da Saúde. Instituto Nacional de Câncer (Inca). Pare de fumar: jovem e tabaco. Rio de Janeiro: Inca; 2004.

11. Moreira LB, Fuchs FD, Moraes RS, Bredemeir M, Cardozo S. Prevalência de tabagismo e fatores associados em área metropolitana da região Sul do Brasil. Rev Saude Publica 1995; 29(1):20-23.

12. Carvalho C. Cresce incidência de câncer da boca no Brasil. Rev Bras Odontol 2003; 60(1):36-39.

13. Brasil. Ministério da Saúde. Pare de fumar: atualidades sobre o tabagismo - curiosidades sobre o tabagismo passivo. Rio de Janeiro: Inca; 2004.

14. Brasil. Ministério da Saúde. Fumantes inveterados têm maior risco de depressão - Pare de fumar: atualidades sobre tabagismo. Rio de Janeiro: Inca; 2007.
15. Brasil. Ministério da Saúde. Fumantes morrem dez anos mais cedo. Rio de Janeiro: Inca; 2004.

16. Doll R, Peto R. The causes of cancer: quantitative estimates of avoidable risks of cancer in the United States today. J Natl Cancer Inst 1981; 66(6):11911308.

17. Brasil. Ministério da Saúde. Secretaria de Atenção à Saúde. Coordenação de Prevenção e Vigilância. A situação do câncer no Brasil. Rio de Janeiro: Inca; 2006.

18. Ribeiro FS, Wünsch Filho V. Avaliação retrospectiva da exposição ocupacional a cancerígenos: abordagem epidemiológica e aplicação em vigilância em saúde. Cad Saude Publica 2004; 20(4):881-890.

19. Brasil. Ministério da Saúde. Fatores ocupacionais. Rio de Janeiro: Inca; 2006.

20. Smith-Warner SA, Spiegelman D, Yaun SS, van den Brandt PA, Folsom AR, Goldbohm RA, Graham S, Holmberg L, Howe GR, Marshall JR, Miller AB, Potter JD, Speizer FE, Willett WC, Wolk A, Hunter DJ. Alcohol and breastcancer in women: a pooled analysis of cohort studies. JAMA 1998; 279:535-540.

21. Bosch FX, Manos MM, Munoz N, Sherman M, Jansen AM. Prevalence of papillomavirus in cervical cancer: a worldwide perspective. J Natl Cancer Inst 1995; 87(11):796-802.

22. Cesar JA, Horta BL, Gomes G, Houlthausen RS, Willrich RM, Kaercher A, Iastrenski FM. Fatores associados à não realização de exame citopatológico de colo uterino no extremo sul do Brasil. Cad Saude Publica 2003; 19(5):1365-1372.

Artigo apresentado em 24/10/2007

Aprovado em 21/05/2008

Versão final apresentada em 06/08/2008 